\title{
Feline Prepucial Urethrostomy
}

\section{Carretto Gustavo Roberto ${ }^{1 *}$, Vitale Verónica², Mangione Mauro ${ }^{3}$, Rizzo Magdalena $^{4}$ and Ugarte Gabriel ${ }^{5}$}

${ }^{1}$ Veterinary, University of Buenos Aires, Faculty of Veterinary Sciences, Surgeon of the Hospital School, Surgery Area, Teacher Catedra de Cirugía for Surgery, Private Practice, Argentina

${ }^{2}$ Veterinarian, University of Buenos Aires, Faculty of Veterinary Sciences, Coordinator of the Vespertino and Surgeon's Shift of the School Hospital, Surgery Area, Teacher Catedra of Surgery, Argentina

${ }^{3}$ Veterinary, University of Buenos Aires, Faculty of Veterinary Sciences, Hospital School, Surgery Intern, Cirugía, Private Practice, Argentina

${ }^{4}$ Veterinary, University of Buenos Aires, Faculty of Veterinary Sciences, Hospital School, Concurrent, Private Practice, Argentina

${ }^{5}$ Veterinary, University of Buenos Aires, Faculty of Veterinary Sciences, Anesthetist of the School Hospital, Private Practice, Argentina

*Corresponding Author: Carretto Gustavo Roberto, Veterinary, University of Buenos Aires, Faculty of Veterinary Sciences, Surgeon of the Hospital School, Surgery Area,

Teacher Catedra de Cirugía for Surgery, Private Practice, Argentina.
Received: March 12, 2020

Published: April 16, 2020

(C) All rights are reserved by Carretto Gustavo

Roberto., et al.

\begin{abstract}
A male feline of 9 years of age with clinical signs of hematuria/dysuria is received and bearing in mind that this is not the first episode it is decided to perform a prep t urethrostomy, This is a permanent surgical procedure, which could be used as an alternative to perineal urethrostomy in felines. It is indicated for the treatment of tumor pathologies, severe trauma, stenosis caused by repeated catheters, relapses of urethral blockages by urinary sediments or lithos.
\end{abstract}

Keywords: Feline; Male; FLUTD; Perineal; Foreskin; Surgery

\section{Introduction}

Feline lower urinary tract disease (FLUTD) actually brings together a group of pathologies affecting the lower urinary tract, cystitis and urethritis, by stones or sediments, with partial or total obstruction of the urethra. It has an incidence of $0.5-4 \%$ in the feline population, and a high tendency to resort. When clinical treatment fails, the surgical (perineal feline urethrostomy) consisting of a mouthful of the pelvic urethra to the skin of the perinose is used. We present as an alternative the feline preputial urethrostomy, which consists of the mouthing of the pelvic urethra, cranial to the bulbourethral glands, to the fundus of the foreskin [1-4].

\section{Materials and Methods}

This little-wide surgical procedure was first presented by veterinary physician Marcelo Catalano, Assistant Professor of the Department of Clinic, General Surgery Area, of the Faculty of Vet- erinary Sciences of the National University of the Center of the Province of Buenos Aires, in the Technical Supplement of the journal the College of Veterinarians of the Province of Buenos Aires No. 56 of October 2013. The following description is based entirely on that procedure.

\section{Case Description and Discussion}

In the Surgery service of the Hospital School of the Faculty of Veterinary Sciences of the University of Buenos Aires, we received a common European feline, male of 9 years of years of age, of 4.5 $\mathrm{kg}$ of weight, with clinical signs of FLUTD, this being its $3^{\text {rd }}$ episode, so we opt for surgical treatment. We perform the tricotomy of the perineal area, base of the tail and the caudal part of the hind limbs. A jar is placed in the anus. The 3 surgical washes are made with $4 \%$ chlorhexidine soapy solution and covered with a sterile cloth. He takes it to the O.R. Place the patient in sternal decubitus in Tren- 
delenburg position and attach the tail to cranial with adhesive fabric. Once positioned, the cloth is removed and embedded with $0.5 \%$ chlorhexidine solution. We catheterized the urethra with a Tomcat-type probe. We make an incision in ventral of the foreskin of full thickness, skin, subcutaneous and prepucial mucosa, in position 6 of the clock, cone scalpel handle no. 3 and leaf no. 11 . We perform is with iris scissors around the base of the penis just at the junction between the prepucial mucosa and the penile mucosa (foreskin fundus). The paraprepucial skin is taken with steering stitches and gently poured to the back. We disseminate the subcutaneous tissue with girl Metzenbaum scissors and does in the left and bulbospongiosus muscles bilaterally of the ischiatic arch until we observe the presence of the bulbourethral glands. On the dorsal of the penis the retractor muscle of the penis and in ventral the ventral ligament of the penis is dissected. We release the union between the pelvic urethra and the base of the pelvis, ensuring with the index finger that the pelvic urethra has been released in its entirety. An incision made, including the urethral mucosa, with scalpel mango no. 3 and sheet 11 from the tip of the pene to the muscles urethralis and bulbospongiosus, using as a guide the Tomcat probe.

Remove the Tomcat probe and use an iris scissors from there to continue the umlaut to cranial to the bulbourethral glands, where the urethra is wider (1.6 - $2 \mathrm{~mm})$. After the umlaut, we place a closed halsted to the joint, in the resulting urethral hole, to verify the correct width. We channel the urethra with a $\mathrm{K} 35$ probe that we will keep for 7 more days after the surgery is complete. We begin to place the points of polydioxanone or polyglycolylate $0-4$, which will attach the urethral mucosa to the mucosa of the foreskin: the first at 12 hs o'clock, the $2^{\text {nd }}$ and $3^{\text {rd }}$ at 11 and at $1,4^{\text {th }}$ and $5^{\text {th }}$ at 10 and 2 . At that point a transfixion ligation with nylon monofilament 2-0 is placed on the penis and amputated by anchoring the stump to the subcutaneous, then we continue with the suture at 9 and 3, 8 and 4, 7 and 5. Finally we sutured the ventral part of the foreskin with nylon monofilament 3-0, where it was influenced at the beginning of the surgery and the ventral skin to it.
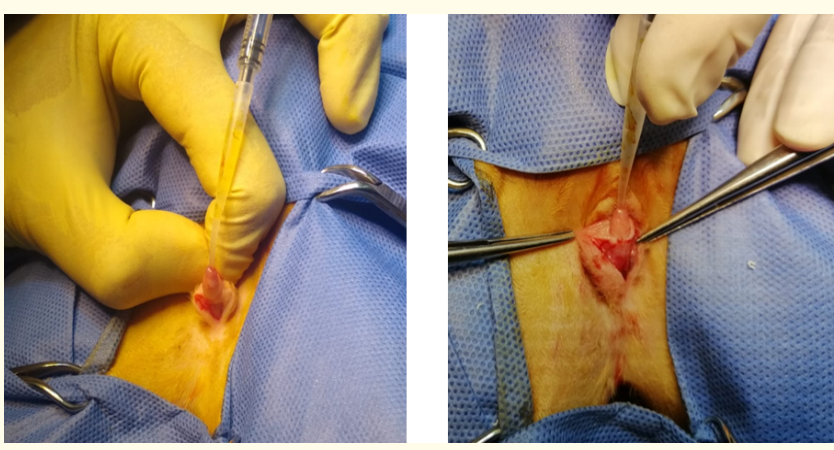
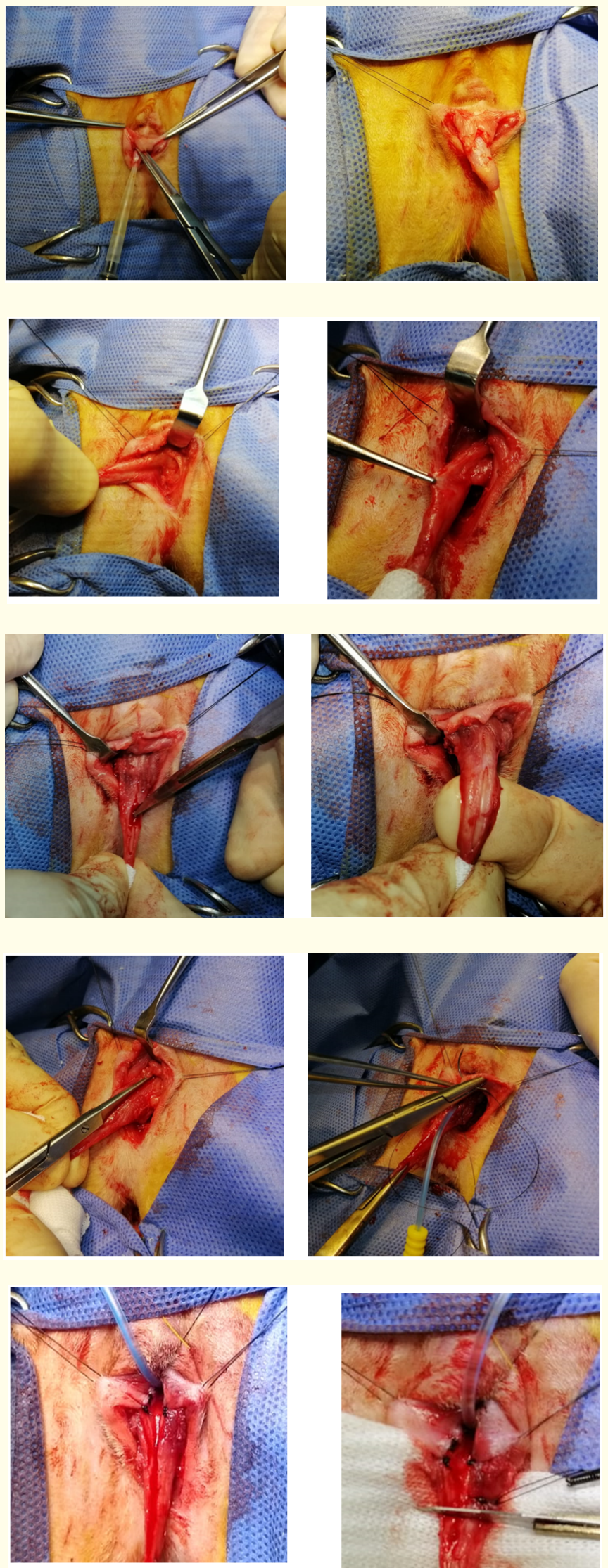

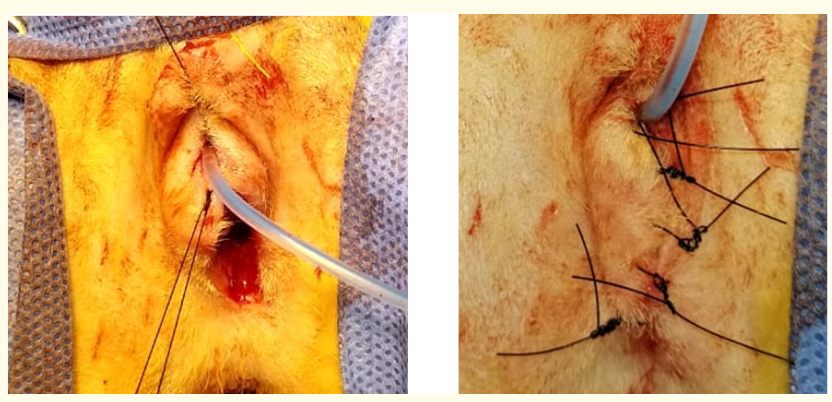

Figure

\section{Conclusion}

Perineal ureterostomy is a widely disseminated palliative surgical procedure that performs well with some possible complications. Stoma stenosis, is the most common and is usually due to the mouthing of the proximal penile urethra instead of the distal pelvic urethra, but also to post-surgical traumatic damage of the urethral mucosa and the advance of the skin on it. Being more exposed also does not prevent upstream bacterial contamination/ infection vía (cystitis and urethritis). The prepucial urethrostomy offers us some advantages, as the urethral mucosa is completely covered and protected, since we link urethral mucosa with prepucial mucosa, the possibility of stenosis and up-linking contamination is minimal.

\section{Bibliography}

1. Bojrab M Joseph. "Current Techniques in Small Animal Surgery". Editorial Intermedica. Buenos Aires. Fourth Edition (2000): 430-434.

2. Catalano Marcelo. "Penean amputation with intraprepucial mouthpiece urethrostomy in the Cat". Technical Supplement of Revista No. 56 of the College of Veterinarians of the Province of Buenos Aires (2013).

3. Fossum Theresa Welch. "Small Animal Surgery". Editorial Intermedica. Buenos Aires (1999): 555-558.

4. Lih-Seng Yeh., et al. "Modified perineal urethrostomy using preputial mucosa in cats". Journal of the American Veterinary Medical Association 216.7 (2000): 1092-1074.

\section{Assets from publication with us}

- Prompt Acknowledgement after receiving the article

- Thorough Double blinded peer review

- Rapid Publication

- Issue of Publication Certificate

- High visibility of your Published work

Website: $\underline{w w w . a c t a s c i e n t i f i c . c o m / ~}$

Submit Article: www.actascientific.com/submission.php Email us: editor@actascientific.com

Contact us: +919182824667 